\title{
Mass and momentum transfer by solitary internal waves in a shelf zone
}

\author{
N. Gavrilov ${ }^{1,2}$, V. Liapidevskii ${ }^{1,2}$, and K. Gavrilova ${ }^{1,2}$ \\ ${ }^{1}$ Lavrentyev Institute of Hydrodynamics, Novosibirsk, 630090, Russia \\ ${ }^{2}$ Novosibirsk State University, Novosibirsk, 630090, Russia \\ Correspondence to: V. Liapidevskii (liapid@hydro.nsc.ru)
}

Received: 11 November 2011 - Revised: 20 January 2012 - Accepted: 6 March 2012 - Published: 3 April 2012

\begin{abstract}
The evolution of large amplitude internal waves propagating towards the shore and more specifically the run up phase over the "swash" zone is considered. The mathematical model describing the generation, interaction, and decaying of solitary internal waves of the second mode in the interlayer is proposed. The exact solution specifying the shape of solitary waves symmetric with respect to the unperturbed interface is constructed. It is shown that, taking into account the friction on interfaces in the mathematical model, it is possible to describe adequately the change in the phase and amplitude characteristics of two solitary waves moving towards each other before and after their interaction. It is demonstrated that propagation of large amplitude solitary internal waves of depression over a shelf could be simulated in laboratory experiments by internal symmetric solitary waves of the second mode.
\end{abstract}

\section{Introduction}

In coastal zones, shoreward propagation of surface and internal waves generally leads to breaking. The turbulence, generated by breaking and mixing processes at the wave fronts, induces the very effective mechanism of energy dissipation and momentum exchange, leading to intensive sediments suspension and transport in the shelf zone. Nonlinear internal waves generated by tides as well as by interaction of flows with topography play the important role in the energy transfer from the large-scale motion to small-scale mixing. The large amplitude internal waves can contain well mixed cores, by which trapped fluid is carried horizontally for a long distance (Maxworthy, 1980; Tung et al., 1982; Stamp and Jacka, 1995; Nash and Moum, 2005; Lamb, 2003; Derzho and Grimshaw, 2007). Propagating to shore, the subsurface internal waves of depression can transform into the large amplitude internal waves of elevation (Helfrich and Melville, 2006; Scotti and Pineda, 2004; Wallace and Wilkinson, 1988; Klymak and Moum, 2003). The transition from wave-like motion to the separate moving soliton-like waves ("solibores") containing trapped core is the common feature of the run up process of internal waves. It can be observed in any shelf zone with high internal wave activity as well as in laboratory experiments. This study is based on the laboratory run up experiments for internal waves. It is shown that an internal solitary wave of elevation was destroyed very quickly during its run up in a "swash" zone along the "dry" bottom, but the presence of a thin dense layer before the wave front prevented the entrainment and mixing processes very effectively. The simple mathematical model describing the nonstationary interaction and decaying of internal solitary waves is suggested.

\section{Laboratory experiments}

Experiments were carried out in a test tank of length $3.2 \mathrm{~m}$, width $0.2 \mathrm{~m}$ and depth $0.35 \mathrm{~m}$, the walls of the test tank were made of Perspex. The test tank was divided by a vertical removable wall in two parts as it is shown in Fig. 1 (line R). The geometrical set-up of the experiments is clear from the sketches shown in Fig. 1. Figure 1a illustrates the experiments on the bottom solitary wave evolution over a slope (run up experiments). Figure $1 \mathrm{~b}$ gives light on the subsurface solitary wave evolutions, and Fig. 1c shows the special installation (inclined bottom and lid in the flume) to provide the shoaling of symmetric solitary waves of the second mode. Details of each experiment will be described below. A weak solution of sugar in water was used to create the stratification. The front propagation speed was evaluated from 


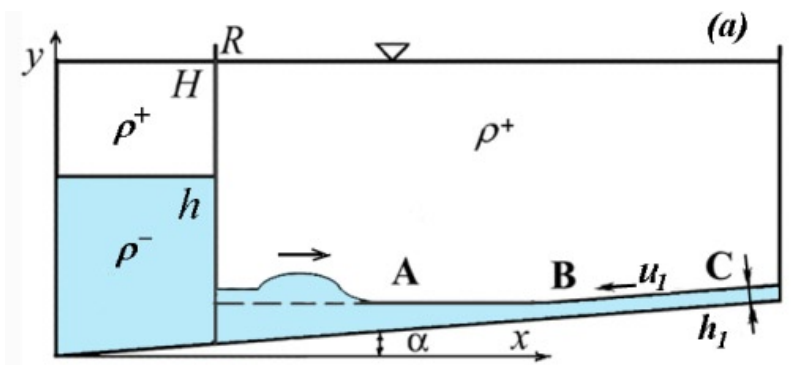

(b)
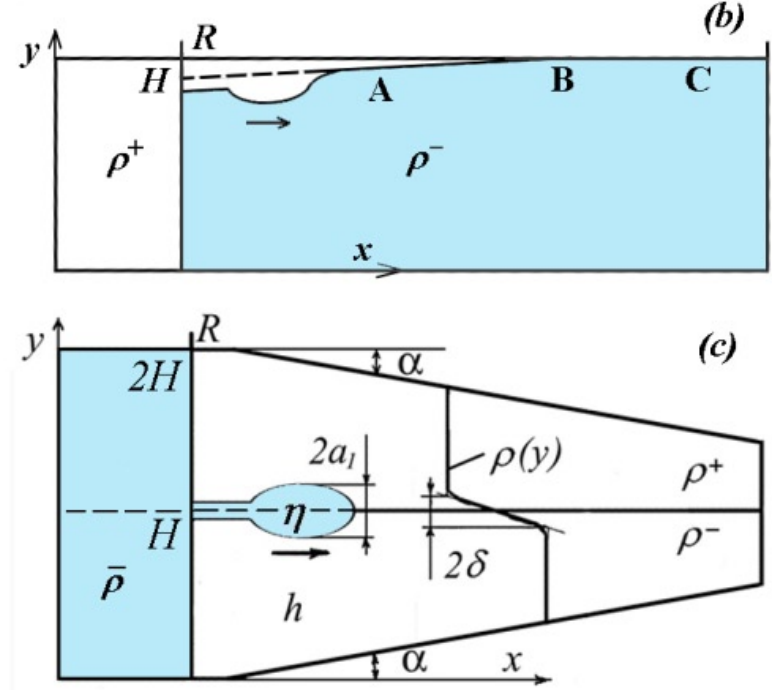

Fig. 1. A sketch of the lock problem in two layer fluid (side view). Internal waves are generated by the removal of slice gates $\mathrm{R}:$ (a) run up of the bottom internal waves; $h_{1}$ is the thickness of a thin layer of dense fluid flowing along the slope; (b) short intrusion along the subsurface layer of light fluid of variable thickness; (c) solitary waves of the second mode at the interface between symmetric homogeneous layers of different density.

video-records obtained by a digital camera at the acquisition rate of 25 frames per second. The flow visualization is portrayed in Fig. 2, where we show photographs of the generated solitary waves against the background of the luminous screen with a grid of inclined lines imposed on it. The optical inhomogeneities related to the perturbation propagation are distinctly seen. In high density gradient regions, we observed the characteristic distortion of lines, whereas the optical transparency of the fluid changes in the mixing areas (Ermanyuk and Gavrilov, 2007). In addition, dense fluid, which propagates along the bottom in the form of a solitary wave, is slightly tinted with an ink solution for visualizing the mass transfer processes. The external wave boundaries are determined by the break and change in thickness of the inclined lines.

In the first series of experiments (Fig. 1a), the lock problem for a two-layer fluid of densities $\rho^{ \pm}$over an inclined bottom was considered $(\tan \alpha=0.012, \alpha$ is the angle of bottom inclination). Two cases are investigated. In the first, the two-layer system was initially at rest. The position "B" in Figs. 1a and 2a corresponds to the intersection of the pycnocline with the bottom. The position " $\mathrm{C}$ " was chosen near the end of the flume where there was no dense fluid at the start of experiment ("dry" bottom). In the second case, the experiment was performed as in the previous case. The only difference was a thin layer of fluid with density $\rho^{-}$, the velocity $u_{1}$ and the thickness $h_{1}$, flowing over the slope up to the position "B". The thickness $h_{1}$ was small enough to provide equilibrium between gravity and friction forces between the positions "B" and "C", so the velocity $u_{1}$ and the thickness $h_{1}$ of the flowing layer were supposed to be constants. After the removal of slice gate, the bore-like structure was gradually transforming into the solitary waves of elevation, which were running up the slope. The photos of the leading solitary wave taken at the corresponding position of the tank are shown in Fig. 2. Between positions "B" and "C" the wave shown in Fig. 2a was running up over the "dry" bottom, since $h_{1}=0$.

The waves shown in Fig. $2 b$ were produced in the same conditions. The only difference is the thin layer of dense fluid $\left(h_{1}=3 \times 10^{-3} \mathrm{~m}, u_{1}=3 \times 10^{-3} \mathrm{~m} \mathrm{~s}^{-1}, \rho_{1}=\rho^{-}\right)$, flowing down the incline and "wetting" the bottom upstream the wave front. One can see that the wave has been destroyed very quickly during its run up along the "dry" bottom (Fig. 2a, "C"), but the existence of a thin layer before the wave front prevented the entrainment and mixing processes very effectively (Fig. 2b, "C"). Therefore, the "wetting" of the bottom by a preceding wave during the run up of an internal wave train can result in the reducing of near bottom turbulence, and in increasing of the "swash zone" of internal waves. It is clear that the reason for such difference between cases (a) and (b) is the bottom friction, which is very sensitive to the thickness of the dense layer at the wave front. Bottom friction has no effect on the propagation of subsurface internal waves in oceans. But in laboratory experiments, the bottom friction effects are replaced by the surface tension effect; and the free surface can be considered as a rigid lid in experiments on large amplitude internal wave propagation. The dependence of solitary wave structure on the initial thickness of the thin upper layer of light fluid in two-layer experiments has been investigated in the second series of experiments (Fig. 1b). Just before the experiment, small amounts of fluid of the density $\rho^{+}$was put near the surface of dense fluid $\left(\rho=\rho^{-}\right)$on the right side of the lock so that the thickness of light layer at rest was varying almost linearly and vanished at the position "B". Then a short intrusion of the light fluid $\left(\rho=\rho^{+}\right)$was generated after removal of slice gate " $R$ ". As in the previous experiments, the intrusion front took the form of a solitary wave moving along the subsurface light layer of variable thickness. The evolution of the front is shown in Fig. 3. At the position "A", it has the form of the solitary wave with the initial layer thickness just before the front $\eta_{\mathrm{A}}=4 \times 10^{-3} \mathrm{~m}$. At the position "B" the thickness of the initial layer vanishes and the intrusion 
(a)

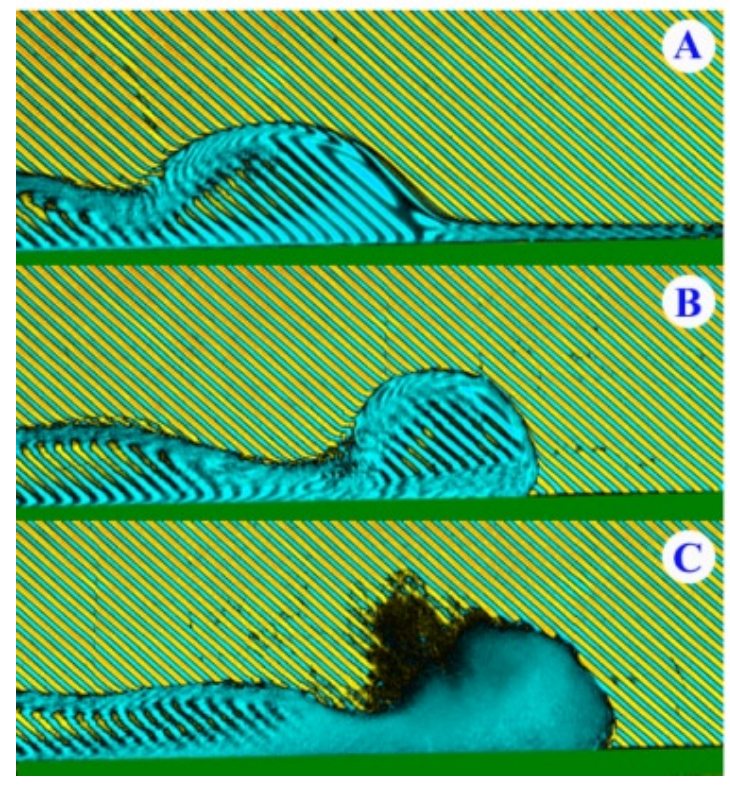

(b)

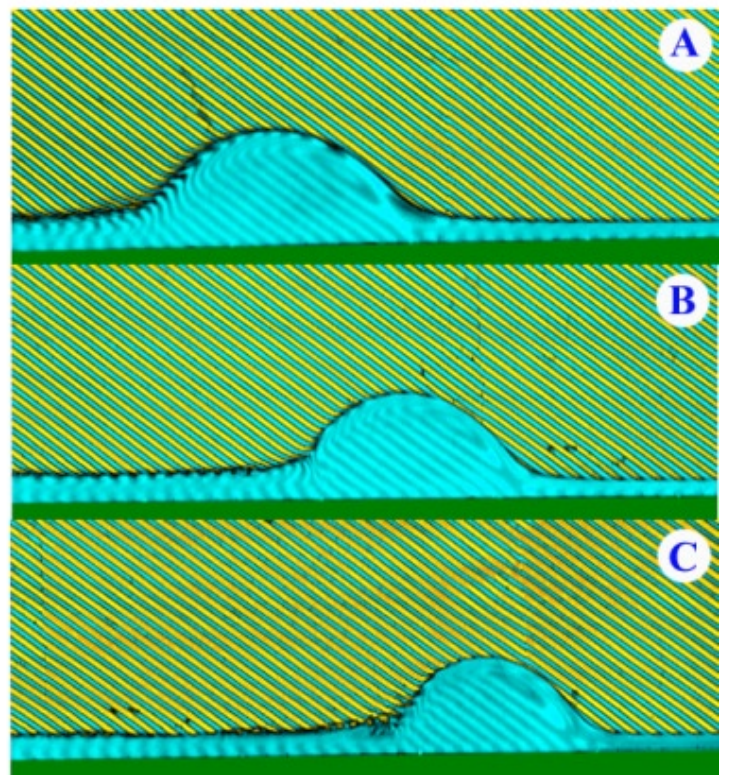

Fig. 2. Run up of the leading solitary wave of elevation along the slope. Capital letters are the positions of the wave in experimental installation (Fig. 1a): (a) $h_{1}=0 ;$ (b) $h_{1}=3 \cdot 10^{-3} \mathrm{~m}, u_{1}=3 \times 10^{-3} \mathrm{~m} \mathrm{~s}^{-1}$.

front transforms into a head of gravity flow (Ermanyuk and Gavrilov, 2007). This head is completely destroyed at the position "C" by the surface friction effects. Comparing Figs. 2a and 3 , we can see that in laboratory scale of flow surface tension and bottom friction affect the gravity current front in a similar way. Therefore, we must take special efforts to simulate large-scale subsurface internal waves in the laboratory conditions. To avoid the bottom and surface effects on large amplitude waves of depression, the third series of laboratory experiments were conducted for short intrusions at the interface of two mixable fluids of different densities (Fig. 1c).

The tank geometry was symmetric relative to the plane $y=H$ with the ordinate $y$ chosen in the vertical direction. So the bottom and the lid of the tank had the same angles of inclination $\alpha$ to the horizon. The length of the compartment with the mixed fluid of density $\bar{\rho}=\left(\rho^{+}+\rho^{-}\right) / 2$ was chosen so that only one solitary wave at the interface was produced. It was also symmetric relative to the undisturbed interface. Due to the flow symmetry, the corresponding component of the Reynolds stress at $y=H$ vanished. The velocities generated in the homogeneous layers by the intrusion were small enough and the friction at the bottom could be neglected too. But the friction between the surrounding fluid and the intrusion should be included in the mathematical model to describe properly the decay rate of solitary waves propagating along the interface. Note that the first two series of experiments (Fig. 1a and b) are performed to demonstrate the sensitivity of the gravity flow structure to the bottom and surface friction effects. Therefore, they give only qualitative description of the problem and show

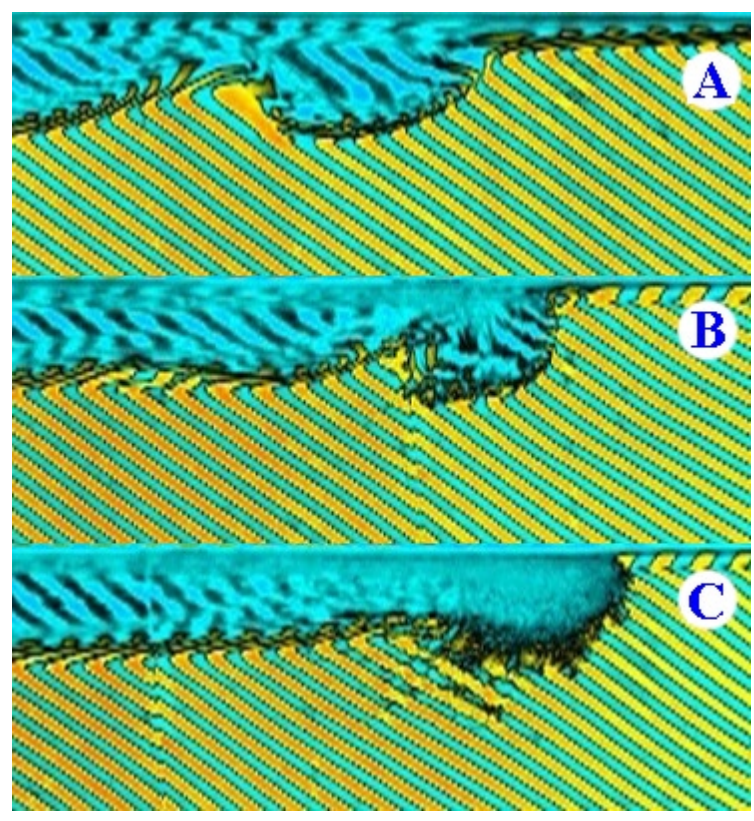

Fig. 3. Transformation of the front of subsurface intrusion: A, B, C are positions of the front corresponding to Fig. $1 \mathrm{~b}$.

that new classes of mathematical models must be developed for adequate simulation of the run up of internal waves in a "swash" zone. So we do not specify in the paper all details of the experiments (the positions "A"-"C", the fluid densities etc). Some approaches to the problem are discussed in Gavrilov and Liapidevskii (2010). As for the third series of 

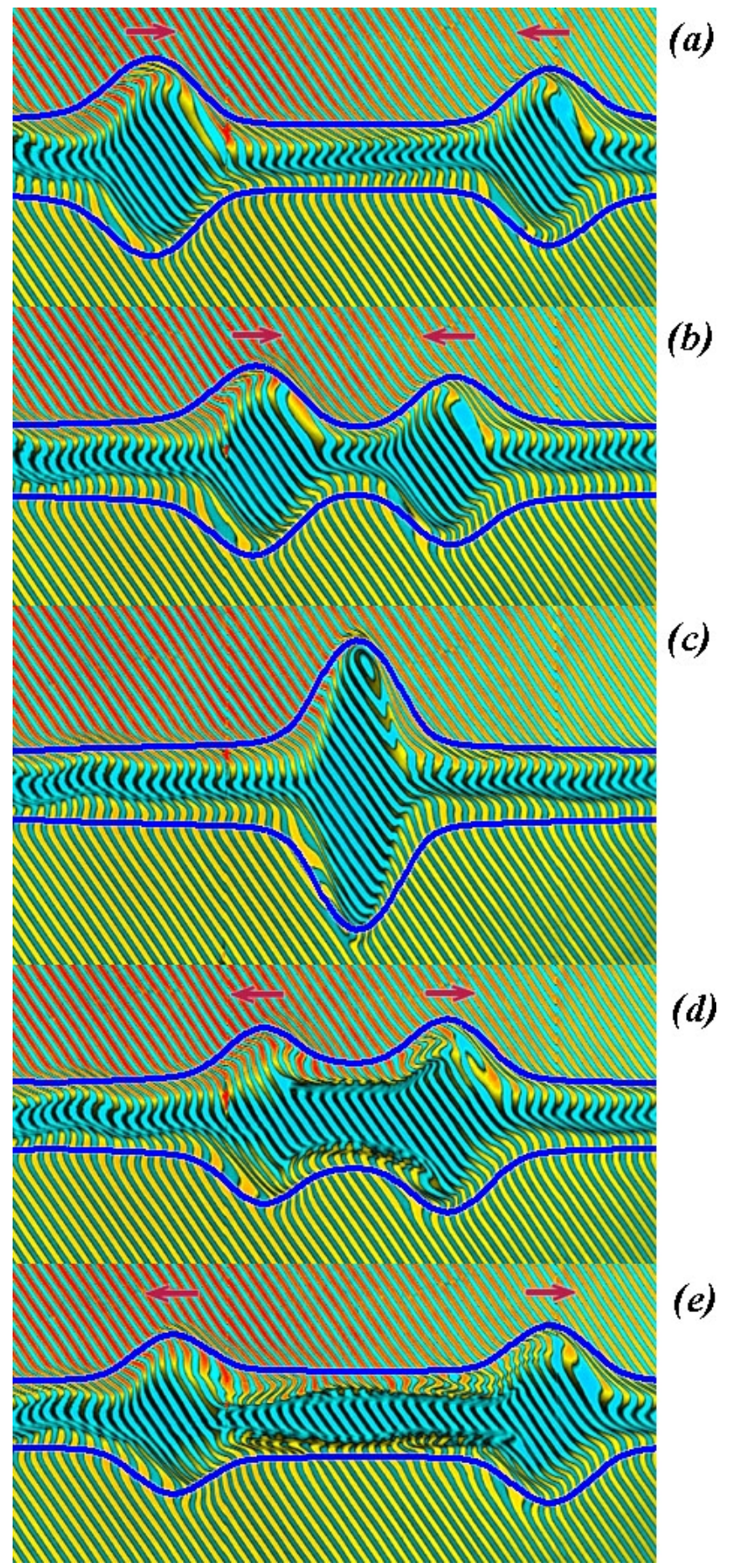

Fig. 4. Interaction of symmetric solitary waves moving toward each other. The time interval between the frames (a-e) is $5 \mathrm{~s}$. The wave parameters are given in the text. The thick (blue) curves show the calculated wave profiles. experiments (Fig. 1c), we will describe in next sections all flow parameters of symmetric internal waves generated experimentally, which are necessary for numerical calculations of the wave evolution and interaction by the mathematical model developed in the following section.

\section{Mathematical model}

There are different approaches to simulating of large amplitude internal waves over a slope. 2-D nonlinear numerical models, taking into account continuous vertical stratification with and without turbulent exchange, have been developed to describe the shoaling solitary internal waves in Vlasenko and Hutter (2002); Lamb (2003). The application of multi-layer shallow water equations with dispersion makes the problem more simple.

Mathematical models using two-layer scheme of flow have been analyzed in Gavrilov et al. (2011). One of them was the full nonlinear model, taking into account the nonhydrostatic effects in both layers (model 1, Choi and Camassa, 1999). The further simplification of the model has been achieved by using the hydrostatic pressure distribution in one of the layers (models 2 and 3 in Gavrilov et al., 2011). It was shown by comparing exact solutions for models $1-3$ that the generation and propagation of large amplitude solitary waves in a two-layer fluid in the channel of finite depth is governed by nonhydrostatic effects in an outer layer. It means that for internal waves of depression the upper layer is supposed to be hydrostatic (model 2) and for waves of elevation the bottom layer can be considered as hydrostatic (model 3$)$.

In Boussinesq approximation $\left(0<\left(\rho^{-}-\rho^{+}\right) / \rho^{+} \ll 1\right)$, the second-mode internal waves are symmetric with respect to the channel midline $y=H$ for the symmetric initial stratification (Fig. 1c). Therefore, it is sufficient to consider only the flow in the bottom half of channel $(0<y<H)$ for describing the symmetric-wave propagation in the interlayer. Let the wave profile (the depth of perturbed homogeneous layer) be represented by $y=h(t, x), u=u(t, x)$ be the mean velocity of the bottom homogeneous layer, $w=w(t, x)$ be the mean velocity of the fluid intrusion, $\eta=\eta(t, x)$ be the half-thickness of the intermediate layer and $g$ be the gravity acceleration. The values $h, \eta$ and $z=z(x)$ are related as follows

$h+\eta+z=H=$ const,

where $z=z(x)$ is the bottom form. In the domain $(0<y<$ $H$ ) symmetric internal waves of the second mode can be considered as the waves of depression, so to describe their dynamics we use the model 2 from Gavrilov et al. (2011). Let $H$ be the reference length, $\bar{b}=\left(\bar{\rho}-\rho^{-}\right) g / \rho^{+}$be the reference buoyancy and $\rho^{+}$be the reference density. In dimensionless variables $\left(H=1, \rho^{+}=1, \bar{b}=1\right)$, the two-layer shallow water equations representing the model 2 (Eq. 16 in Gavrilov et al., 2011) take the form 


$$
\begin{aligned}
& h_{t}+(h u)_{x}=0, \\
& \eta_{t}+(\eta w)_{x}=0, \\
& u_{t}+u u_{x}+h_{x}+z_{x}+p_{x}+\frac{\beta}{3 h}\left(h^{2} \frac{d^{2} h}{\mathrm{~d} t^{2}}\right)_{x}=f^{-}, \\
& w_{t}+w w_{x}+p_{x}=f^{+}, \\
& f^{-}=-c \frac{(u-w)|u-w|}{h}, \quad f^{+}=c \frac{(u-w)|u-w|}{\eta}, \\
& \frac{d}{\mathrm{~d} t}=\frac{\partial}{\partial t}+u \frac{\partial}{\partial x},
\end{aligned}
$$

where $p$ is the dimensionless pressure at $y=H$. Note that the total flow rate $Q=h u+\eta w=0$, so we can exclude the variables $p, \eta=1-h-z$ and $w=-h u /(1-h-z)$ from (1). After that, Eq. (1) is reduced to the system of two equations for the variables $h$ and $u$, which is the analog of the GreenNaghdi equations (Green and Naghdi, 1976), describing nonlinear waves in two-layer fluids. The dimensionless value $\beta$ is just a scaling coefficient between vertical and horizontal axes and it can be excluded from the system by the dilatation of independent variables. The only empiric constant in Eq. (1) is the friction coefficient $c$. It is shown in Gavrilov et al. (2011) that the numerical values of $c=0.01$ and $\beta=0.5$ give satisfactory results for numerical calculation of solitary waves dynamics. So we have used these values for calculations below.

The advantage of Eq. (1) is that the simple exact solutions describing the solitary waves can be constructed in explicit form. Let the dissipative processes be ignored, and the bottom be horizontal, i.e. $c=0$ and $z_{x} \equiv 0$. Consider traveling waves running in the two-layer fluid at rest, i.e. the solutions of Eq. (1), which depend on the variable $\xi=x-D t$, $(D \equiv$ const). Suppose that the following conditions are satisfied for $|\xi| \rightarrow \infty$

$h \rightarrow h_{0}, \quad h^{\prime} \rightarrow 0, \quad h^{\prime \prime} \rightarrow 0$

(here and below, the prime denotes differentiation with respect to the variable $\xi)$. For given $h_{0}\left(0<h_{0}<1\right)$, the traveling wave satisfying Eq. (2) represents a soliton and depends on the Froude number $F r=D / \sqrt{\bar{b} H}$. Note that in the dimensionless variables $F r=D$, where $D$ is the dimensionless velocity of the traveling wave. It is shown in Gavrilov et al. (2011) that the profile of a solitary wave $h=h(\xi)$ can be found by quadrature

$\xi=\xi(h)= \pm \int_{h_{0}}^{h} \frac{d s}{\sqrt{G(s)}}$,

where

$\left(h^{\prime}\right)^{2}=G(h)=\frac{3\left(h-h_{0}\right)^{2}\left(F r^{2}-h+h^{2}\right)}{\beta F r^{2} h_{0}^{2}(1-h)}$.

It follows from Eq. (4) that the admissible interval for $h$ is $h_{m} \leq h<h_{0}<1$ and $F r \leq 1 / 2$. Here $h_{\mathrm{m}}=\frac{1+\sqrt{1-4 F r^{2}}}{2}$

is the minimal depth of the bottom layer in the wave and $A=1-h_{\mathrm{m}}$ is the (dimensionless) amplitude of the solitary wave. Note also that the limiting case $h_{0} \rightarrow 1$, when the solitary wave propagating along the zero-thickness interface between the homogeneous layers of different densities, can be easily found from Eqs. (3)-(4) (see Gavrilov et al., 2011). The constructed above solution is used below as initial data in nonstationary calculation of solitary wave shoaling and interaction. The velocity distribution in a solitary wave can be found from relations:

$h(D-u)=h_{0} D, \quad(1-h)(D-w)=\left(1-h_{0}\right) D$.

In a real system with dissipation, which can be a stratified fluid, solitary waves propagating with a constant velocity cannot be realized. During the propagation, the solitary wave loses mass and its velocity decreases. Nevertheless, the motion of an internal wave is quasi-steady: at each moment of time, its shape coincides with that of the stationary wave of the same amplitude. The discrepancies are observed only at the small portion adjoining to the area of flow behind the wave.

The problem regarding the interaction of the solitary waves moving toward each other can be solved numerically as the Cauchy problem for Eq. (1), with the initial data in the form of solitary waves Eqs. (3)-(5) propagating with the velocities $D_{1}>0$ and $D_{2}<0$ and starting to move at a sufficient distance $L_{0}$ so that the initial stratified fluid between them is unperturbed. The numerical algorithm for Eq. (1) is discussed in Gavrilov et al. (2011). It is based on numerical scheme developed for the Green-Naghdi equations in Le Metayer et al. (2010). In Fig. 4 we show the main stages of interaction between the solitary waves realized experimentally.

Reference scales used in experiments on solitary wave interaction are $H=6 \times 10^{-2} \mathrm{~m},\left(\rho^{-}-\rho^{+}\right) g / 2 \rho^{+}=2.5 \times$ $10^{-2} \mathrm{~m} / \mathrm{s}^{-2}, \rho^{+}=10^{3} \mathrm{~kg} \mathrm{~m}^{-3}$. A solitary wave propagating on the left $\left(D_{2}<0\right)$ was produced analogously to the solitary wave moving on the right (Fig. 1c, $\alpha=0$ ). The consecutive positions of solitary waves realized experimentally are shown in Fig. 4a-e through the regular time interval $(5 \mathrm{~s})$.

As initial data for numerical calculations in Eq. (1), we have chosen the following dimensional values: solitary wave amplitudes $A_{1}=2.3 \times 10^{-2} \mathrm{~m}, A_{2}=2 \times 10^{-2} \mathrm{~m}$, the distance between their crests $L_{0}=0.3 \mathrm{~m}$, the half-thickness of the intermediate layer $\eta_{0}=H-h_{0}=9 \times 10^{-3} \mathrm{~m}$. Note that $\bar{b}=\left(\rho^{-}-\rho^{+}\right) g / 2 \rho^{+}$gives the mean buoyancy for the model (1) only for step-wise density distribution in the intermediate layer. For real initial density distribution $\rho=$ $\rho(y)$, which is symmetric relative to the channel midline $y=H$, the mean value $\bar{b}$ varies from $\bar{b}=\left(\rho^{-}-\rho^{+}\right) g / 4 \rho^{+}$ for the linear stratification in the intermediate layer up to $\bar{b}=\left(\rho^{-}-\rho^{+}\right) g / 2 \rho^{+}$for the case of step-wise stratification. 


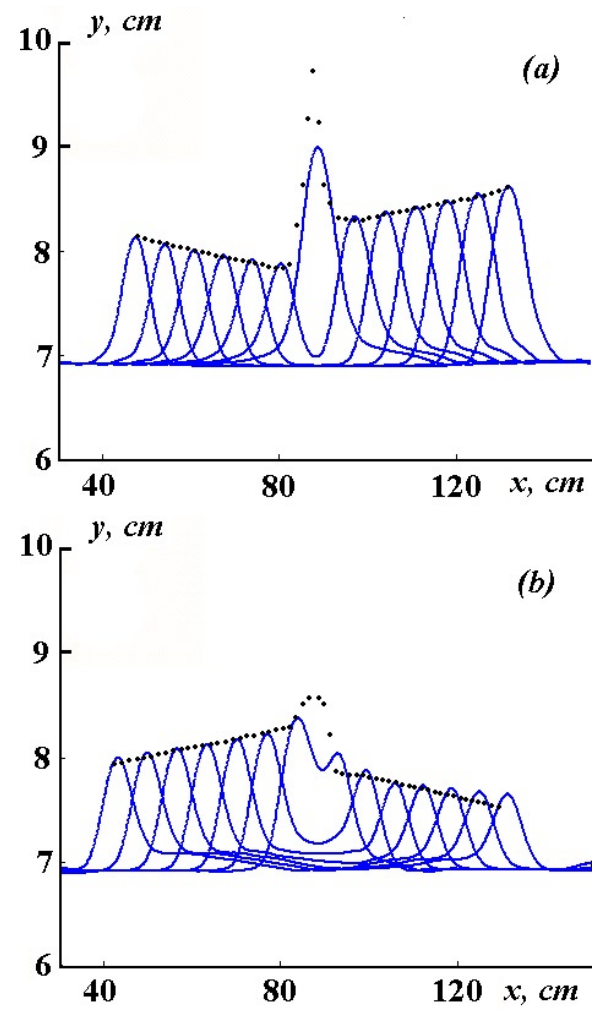

Fig. 5. The decay of symmetric solitary waves moving toward each other. Bold points represent the experimental positions of wave crests through the regular time interval (1 s). Solid lines show the calculated wave profiles through the fixed time interval $(4 \mathrm{~s})$ : (a) waves before interaction $(t<24 \mathrm{~s})$; (b) waves after interaction $(t>24 \mathrm{~s})$.

In our calculations we put $\bar{b}=2.2 \times 10^{-2} \mathrm{~m} / \mathrm{s}^{-2}$. The thick (blue) curves in Fig. 4a-e show the calculated wave profiles. One can see that the numerical solution gives the satisfactory description of the outer boundaries of perturbations in stratified fluid realized experimentally.

The dissipative effects on solitary waves propagation and interaction are demonstrated in Fig. 5. The problem under consideration is the same as those discussed above. The bold points indicate the wave-crest locations found experimentally through the regular intervals of time $(1 \mathrm{~s})$. The reference values are the same as in previous case (Fig. 4). The initial data are: $A_{1}=2.13 \times 10^{-2} \mathrm{~m}, A_{2}=2.6 \times 10^{-2} \mathrm{~m}$, $L_{0}=0.84 \mathrm{~m}, \eta_{0}=9 \times 10^{-3} \mathrm{~m}$. The wave profiles are shown in Fig. 5a through the time intervals $4 \mathrm{~s}$ up to the moment of the waves collision $\left(t_{\mathrm{c}}=24 \mathrm{~s}\right)$, and the wave profiles through the same time intervals are shown in Fig. $5 \mathrm{~b}$ for $t>t_{c}$.

It is seen from Fig. 5 that the model (1) with the numerical value of the friction coefficient $c=0.01$ qualitatively and quantitatively reflects the changes in the phase and amplitude characteristics of solitary waves before and after their interaction. One can also conclude from this figure that the dissipative effects result in the consecutive wave transformation in a nonsymmetric form.
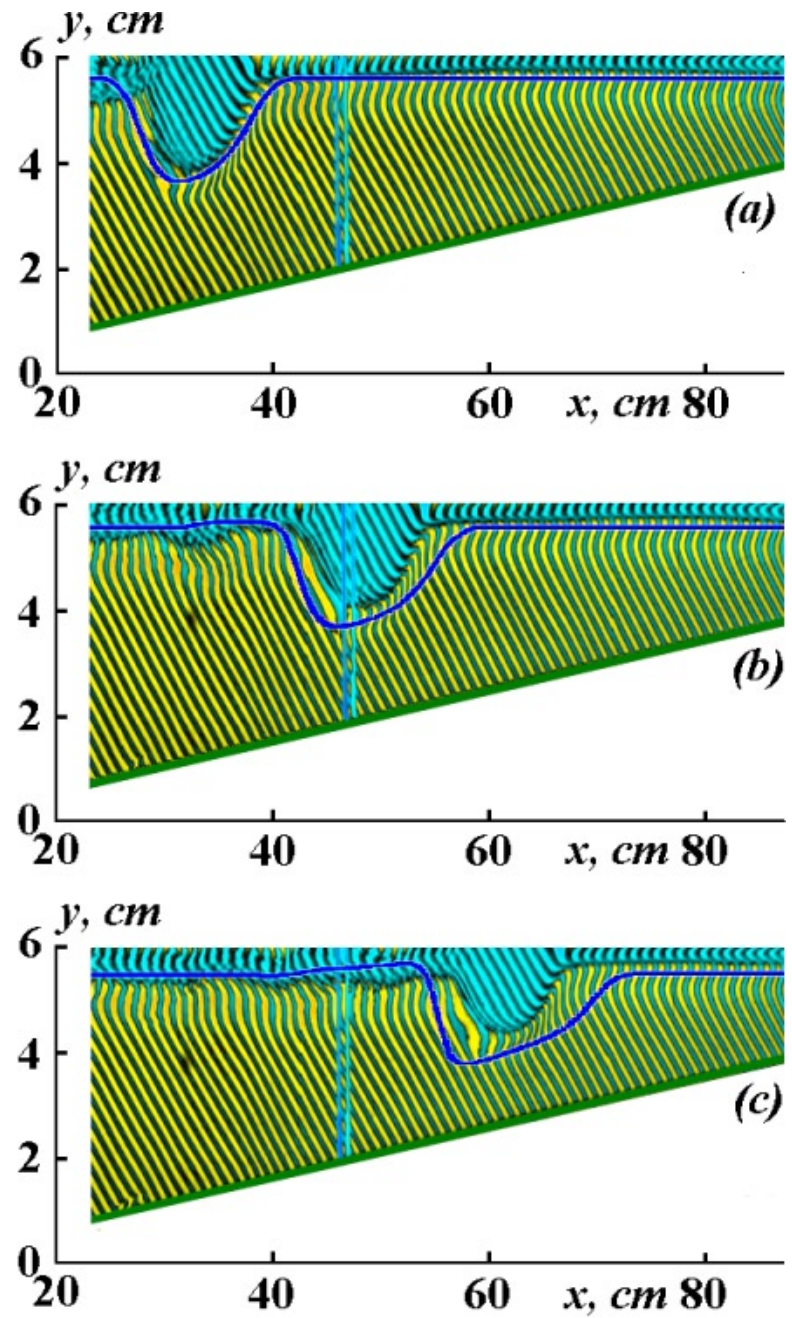

Fig. 6. Shoaling solitary wave $(\tan \alpha=0.05)$. Photos of experiment II (background) taken through the time interval $10 \mathrm{~s}$ are compared with numerical calculations by (1). The lower part of the experimental installation (Fig. 1c, $0 \leq y \leq H$ ) is shown.

\section{Propagation of surface solitary waves over a slope}

The experiments on solitary wave propagations over an incline $(\alpha>0)$ give light on the shoreward mass and momentum transfer by large amplitude subsurface internal waves. Such waves were detected in shelf zones of different seas (Helfrich and Melville, 2006). The evolution of a symmetric solitary wave, which has been produced as sketched in Fig. 1c, is shown in Fig. 6 with the time period between the frames $\Delta t=10 \mathrm{~s}$ (only the lower part of flow $z(x)<y<H$, $z_{x}=\alpha=0.05$ is displayed). The bold (blue) curves show the wave profiles calculated by Eq. (1) for corresponding time. The reference values $H, \bar{b}, \rho^{+}$are the same as in previous sections, the wave moves from the left to the right. All necessary geometric wave parameters can be taken directly from Fig. 6a-c. 
One can see in Fig. 6 that the shoaling internal wave loses its symmetry when the wave amplitude reaches the halfthickness of the flume. The rear part of the soliton sharpens and the back front of the wave takes the form of an internal bore. The model doesn't take into account the mixing in the intermediate layer, so the agreement between numerical and experimental data at this stage of flow (Fig. 6c) is not so satisfactory as at more earlier phases of the wave propagation (Fig. 6a and b).

The ability of the model (1) to simulate the shoaling surface internal waves up to the moment of an internal bore formation and subsequent breaking is demonstrated in Fig. 7 , where the solitary internal wave moving to the left over a slope is shown. Figure $7 \mathrm{a}$ and $\mathrm{b}$ is taken from Vlasenko and Hutter (2002) (Fig. 2a and b). They represent the results of numerical calculation of a shoaling wave in continuously stratified fluid at rest over a slope. The thin lines show the deformation of the density level with the density contour interval of $0.5 \mathrm{~kg} \mathrm{~s}^{-3}$. The 2-D nonlinear numerical model with continuous vertical stratification and turbulent exchange has been developed in Vlasenko and Hutter (2002) to study breaking processes in nonlinear internal waves over a topography. The model contains a number of parameters and have been applied for different types of stratified flows. To calculate the problem under consideration by the model (1), we use the following reference values: $\bar{b}=6 \times 10^{-2} \mathrm{~m} / \mathrm{s}^{-2}$, $H=300 \mathrm{~m}, \rho^{+}=10^{3} \mathrm{~kg} \mathrm{~m}^{-3}$. Initial depth of the upper homogeneous layer was taken as $\eta_{0}=50 \mathrm{~m}$.

The exact solution (3)-(4) with appropriate amplitude was used as initial data for Eq. (1). Thick (blue) curves represent the numerical solutions of Eq. (1), corresponding to the numerical solutions, which were constructed in Vlasenko and Hutter (2002). We can see that the nonstationary numerical solution of Eq. (1) reproduces the characteristic features of the shoaling wave with reasonable accuracy, despite of the two-layer approximation of the flow. Note also that in contrast to the 2-D model of stratified flow developed in Vlasenko and Hutter (2002), the initial data for the model (1) do not generate the tail waves, since we use the exact solitonlike solution of Eq. (1) as the initial data.

\section{Conclusions}

Laboratory experiments described in the paper show that the bottom friction and the surface tension affect strongly the dynamic of large amplitude internal waves. To simulate the propagation of large-scale internal waves in oceans, where the surface tension has no effect, the special geometry of flow has been used (Fig. 1c). It is demonstrated that the problems on shoaling and interactions of solitary internal waves of depression could be represented in laboratory experiments by internal symmetric solitary waves of the second mode (Figs. 4-6).

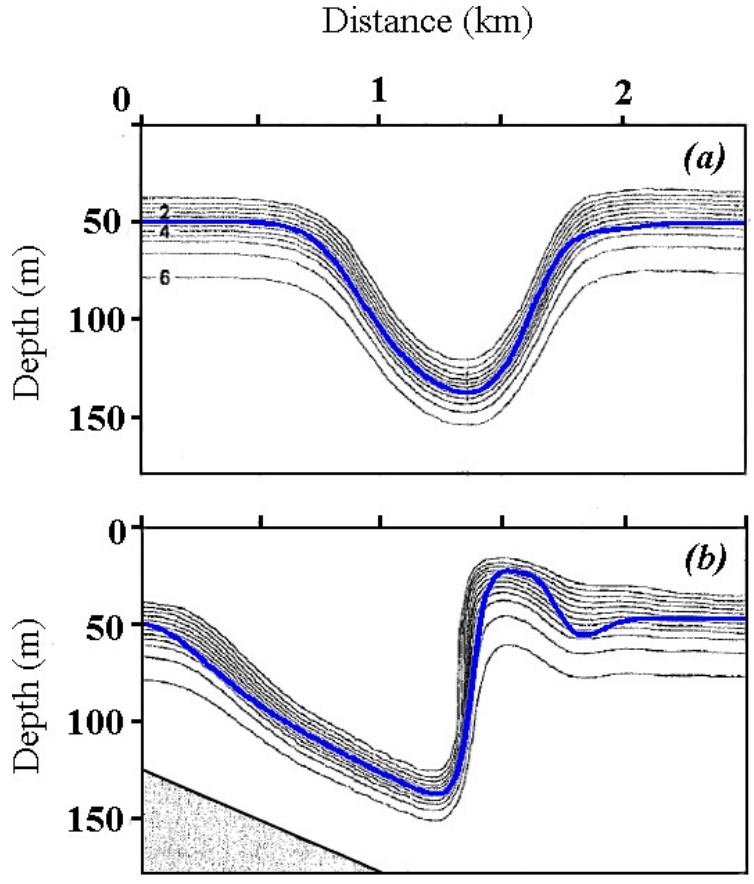

Fig. 7. Shoaling internal wave over a slope $(\tan \alpha=0.05)$. The wave moves to the left. Comparison of numerical results from Vlasenko and Hutter (2002) (thin lines, Fig. 2a and b) with the numerical solution of (1) (bold line). Corresponding wave parameters are given in the text.

The aim of the paper is to develop the simplest mathematical model, which would be capable to reproduce the main features of nonlinear internal waves. It is shown that Eq. (1), which are the two-layer shallow water equations, taking into account the dispersion effects in the bottom layer, can describe large amplitude internal wave evolution in a coastal zone. The equations are the direct extension of the Green-Naghdi equations developed for open channel flows of two-layer stratified fluids. Therefore, exact soliton-like solutions and numerous numerical methods constructed for Green-Naghdi equations could be applied to near shore dynamics of internal waves. Note also, that the solutions of Eq. (1), describing the internal shoaling waves, are compatible with numerical solutions of more complicated models (Fig. 7).

Concerning the run up problem for internal waves, it is demonstrated in laboratory experiments that the structure of an internal wave of elevation in the "swash zone" depends drastically on the thickness of the dense bottom layer; and the "wetting" of the bottom by a preceding wave during run up process can result in the effective reducing of near bottom turbulence (Fig. 2). To describe such phenomenon, new mathematical models, taking into account turbulent mixing at the wave front, must be developed. 
Acknowledgements. The authors are grateful to the referees for their constructive comments. This work was supported by the Russian Foundation for Basic Research (Grants No. 10-01-00338, 12-01-00671), Integration project of the Siberian Division of the Russian Academy of Sciences No. 15.

Edited by: E. Pelinovsky

Reviewed by: two anonymous referees

\section{References}

Choi, W. and Camassa, R.: Fully nonlinear internal waves in a twofluid system, J. Fluid Mech., 386, 1-36, 1999.

Derzho, O. G. and Grimshaw, R.: Assymetric internal solitary waves with a trapped core in deep fluids, Phys. Fluids, 19, 096601, doi:10.1063/1.2768507, 2007.

Ermanyuk, E. V. and Gavrilov, N. V.: A note on the propagation speed of a weakly dissipative gravity current, J. Fluid Mech., 574, 393-403, 2007.

Gavrilov, N. V. and Liapidevskii, V. Yu.: Finite-amplitude solitary waves in a two layer fluid, J. Appl. Mech. Tech. Phys., 51, 471$481,2010$.

Gavrilov, N. V., Liapidevskii, V., and Gavrilova, K.: Large amplitude internal solitary waves over a shelf, Nat. Hazards Earth Syst. Sci., 11, 17-25, doi:10.5194/nhess-11-17-2011, 2011.

Green, A. E. and Naghdi, P. M.: A derivation of equations for wave propagation in water of variable depth, J. Fluid Mech., 78, 237246, 1976.

Helfrich, K. R. and Melville, W. K.: Long nonlinear internal waves, Annu. Rev. Fluid Mech., 38, 395-425, 2006.
Klymak, J. M. and Moum, J. N.: Internal solitary waves of elevation advancing on a shoaling shelf, Geophys. Res. Lett., 30, 2045, doi:10.1029/2003GL017706, 2003.

Lamb, K. G.: Shoaling solitary internal waves:on a criterion for the formation of waves with trapped cores, J. Fluid Mech., 478, 81100, 2003.

Le Metayer, O., Gavrilyuk, S., and Hank, S.: A numerical scheme for the Green-Naghdi model, J. Comput. Phys., 229, 2034-2045, 2010.

Liapidevskii, V. Yu. and Teshukov, V. M.: Mathematical Models of Long-Wave Propagation in an Inhomogeneous Fluid, Izd. Sib. Otd. Ross. Akad. Nauk, Novosibirsk, 2000 (in Russian).

Maxworthy, T.: On the formation of nonlinear internal waves from the gravity collapse of mixing regions in two and three dimensions, J. Fluid Mech., 96, 47-64, 1980.

Nash, J. D. and Moum, J. N.: River plumes as a source of largeamplitude internal waves in the coastal ocean, Nature, 437, 400403, doi:10.1038/nature03936, 2005.

Scotti, A. and Pineda, J.: Observation of very large and steep internal waves of elevation near the Massachusetts coast, Geophys. Res. Lett., 31, L22307, 1-5, doi:10.1029/2004GL021052, 2004.

Stamp, A. P. and Jacka, M.: Deep-water internal solitary waves, J. Fluid Mech., 305, 347-371, 1995.

Tung, K.-K., Chan, T. F., and Kubota, T.: Large amplitude internal waves of permanent form, Stud. Appl. Math., 66, 1-44, 1982.

Vlasenko, V. and Hutter, K.: Numerical experiments on the breaking of solitary internal waves over a slope-shelf topography, J. Phys. Oceanography, 32, 1779-1793, 2002.

Wallace, B. C. and Wilkinson D. L.: Run up of internal waves on a gentle slope, J. Fluid Mech., 191, 419-442, 1988. 